\title{
Producción científica en Scopus de la Universidad de San Martín de Porres, Lima, Perú
}

Álvaro Taype-Rondán ${ }^{1,2, a}$, Laura Luque Bustamante ${ }^{1,2, a}$

\section{RESUMEN}

Objetivo: Analizar la producción científica de la Universidad de San Martín de Porres (USMP) en Scopus.

Material y Métodos: Se realizó un estudio bibliométrico, analizando los artículos publicados en la base de datos Scopus que tengan al menos un autor con filiación de la USMP, evaluando las siguientes variables: Facultad de la USMP involucrada, revista en el que fue publicado, año correspondiente a la publicación, idioma, autoría corresponsal, tipo de artículo, colaboración, colaboración internacional y nombre de autor(es) con filiación USMP.

Resultados: Se hallaron un total de 92 artículos que cumplieron los criterios de inclusión. Se evidenció una tendencia al alza en el número anual de publicaciones. La mayor parte de los artículos $(62,0 \%)$ fueron clasificados como pertenecientes a la Facultad de Medicina Humana. 69,6\% de los artículos, fueron considerados "artículos de colaboración", y 40,2\% fueron considerados "artículos de colaboración internacional", con gran participación de USA y España. Se encontraron 118 autores diferentes con filiación USMP, los cuales publicaron en 70 revistas diferentes (dos de ellas peruanas).

Conclusión: Se evidenció una tendencia al alza en el número anual de publicaciones, por lo que se debería continuar incentivando la producción científica en investigadores, docentes y estudiantes. (Horiz Med 2014; 14(4): 37-42)

Palabras clave: Bibliometría, apoyo a la investigación como asunto, docentes. (Fuente: DeCS BIREME).

\section{Scientific production in Scopus of the University of San Martín de Porres, Lima, Peru}

\section{ABSTRACT}

Objective: To analyze scientific production in Scopus database produced by the University of San Martin dePorres (USMP). Material and Methods: A bibliometric study was performed by analyzing the articles published in the Scopus database with at least one author with USMP affiliation. The following variables were taken in account: USMP school involved, journal in which article was published, year of publication, language, Corresponding author, Publication type, National and international collaboration, number and authors with USMP affiliation.

Results: We found a total of 92 articles with our inclusion criteria. We found a rising trend towards a higher number of publications per year. The majority of articles (62.0\%) belonged to the School of Medicine of the USMP. Likewise, $69.6 \%$ of articles were considered "Collaboration articles and, $40.2 \%$ were established "International collaboration articles", with a significant participation of USA and Spain. We found 118 different authors with USMP affiliation that published in 70 different journals (including 2 Peruvian Journals).

Conclusion: An upward trend in the annual number of publications showed so should continue encouraging the scientific production of researchers, teachers and students. (Horiz Med 2014; 14(4): 37-42)

Key words: Bibliometrics, research support as topic; faculty. (Source: MeSH NLM).

\footnotetext{
Facultad de Medicina Humana, Universidad de San Martín de Porres, Lima, Perú

Massachusetts General Hospital, Boston, MA - EEUU

Médico-Cirujano
} 


\section{INTRODUCCIÓN}

Las universidades, son consideradas centros del saber. Tienen entre sus principales objetivos la ideación y ejecución de investigaciones que permitan generar conocimientos útiles para el desarrollo sostenible de su país(1,2). Sin embargo, muchas universidades latinoamericanas aún no han desarrollado una sólida cultura de investigación (3), problemática en la que está incluido el Perú (4-6).

Actualmente, la producción científica de las universidades es medida por el número de artículos científicos que estas instituciones logran publicar en ciertas bases de datos (7), siendo Scopus una de las bases más utilizadas para este fin, debido a la gran variedad de áreas del conocimiento que abarca, la calidad de sus revistas y los continuos análisis bibliométricos sobre producción científica que se realizan en esta base (8). De tal manera que varios rankings universitarios califican las universidades de acuerdo a su producción científica en Scopus, como son el Quacquarelli Symonds World University Rankings (9), Ranking web of Universities (10), América Economía (11), entre otros.

Una evaluación de la producción científica de las universidades peruanas en Scopus en el periodo 2008-2012, encontró que la universidad con mayor producción científica fue la Universidad Peruana Cayetano Heredia, seguida de la Universidad Nacional Mayor de San Marcos.

En este ranking, encontramos en noveno lugar a la Universidad de San Martín de Porres (3).

La Universidad de San Martin de Porres (USMP) fue fundada en el año 1962. Actualmente, cuenta con nueve facultades que ofrecen dieciocho carreras profesionales (12).

Su Facultad de Medicina Humana, inició sus actividades académicas en 1984 (13), es una de las escuelas de Medicina con mayor número de médicos titulados cada año a nivel nacional.

La investigación es una función básica y obligatoria en la USMP, como se menciona en su Reglamento Interno (14). Por ello, es necesario realizar evaluaciones periódicas de la producción científica de sus Facultades, lo cual permitirá mejorar las estrategias utilizadas para fortalecer la investigación en la USMP. Por ello, el objetivo del presente estudio fue analizar la producción científica de la USMP en Scopus.

\section{MATERIAL Y MÉTODOS}

Se realizó un estudio bibliométrico, analizando los artículos publicados hasta el año 2013 en revistas científicas indexadas a la base de datos Scopus que tengan al menos un autor con filiación de la USMP.

Durante el mes de Marzo del año 2014, cada uno de los autores, elaboró una lista de publicaciones, mediante la realización de dos búsquedas en la base de datos Scopus. En la primera búsqueda, se utilizó el Affiliation Identifier de Scopus para ubicar todos los artículos agrupados bajo la filiación institucional de la Universidad de San Martin de Porres.

Para la segunda, se utilizó el buscador de artículos de Scopus, con las palabras clave: San Martín de Porres Perú.

Posteriormente, se comparó ambas listas para evaluar las discrepancias, que fueron discutidas entre los autores hasta llegar a un concenso. A continuación, se eliminaron los registros duplicados, y se accedió a cada documento para verificar que se tratara de una publicación en una revista científica y que alguno de los autores tenga como filiación a la USMP, eliminando los documentos que no cumplieran con ambos criterios.

Una vez que se obtuvo el listado final de artículos, se tabularon las siguientes variables para cada artículo: Facultad de la USMP involucrada, revista en el que fue publicado, año correspondiente a la publicación, idioma, autoría corresponsal, tipo de artículo, colaboración, colaboración internacional y nombre de autor(es) con filiación USMP.

La "Facultad de la USMP involucrada" fue obtenida al revisar las filiaciones de los autores, o al realizar una búsqueda de los autores implicados para determinar a qué Facultad de la USMP pertenecían. 
De no lograr encontrar estos datos, se clasificó de acuerdo al área del conocimiento al que pertenecía el tema central de la publicación.

Se denominó "artículo de colaboración", a aquel en el cual participó al menos un autor con filiación institucional diferente a la USMP. Se denominó "artículo de colaboración internacional" a aquel en el cual participó al menos un autor con filiación institucional extranjera.

Los datos fueron tabulados en el programa Microsoft Excel 2010. Se realizó el análisis descriptivo, presentando las variables cuantitativas mediante medidas de tendencia central, y las variables cualitativas mediante porcentajes. Para ayudar la visualización de los datos, se utilizaron tablas y figuras.

\section{RESULTADOS}

Se halló, un total de 92 artículos publicados en revistas científicas por la Universidad de San Martín de Porres en Scopus, siendo el más antiguo publicado en el año 1995.

Se evidenció una irregular tendencia al alza en el número anual de publicaciones. La mayor parte de los artículos (62,0\%), fueron clasificados como pertenecientes a la Facultad de Medicina Humana.

El mayor número de publicaciones se observó en el año 2013, seguido del año 2010. La Facultad de Economía, no publicó artículos en los años 2012 y 2013, como se muestra en la Figura 1.

Tabla 1.- Características de los artículos publicados por cada Facultad de la Universidad de San Martín de Porres en Scopus, 1995-2013 $(\mathrm{N}=92)$.

$\begin{array}{lcccccc} & \text { Medicina } & \text { Psicología } & \text { Odontología } & \text { Economía } & \text { Otras } & \text { Total } \\ \begin{array}{l}\text { Total de } \\ \text { publicaciones }\end{array} & 57 & 15 & 6 & 6 & 8 & 92 \\ \begin{array}{l}\text { Autor corresponsal } \\ \text { USMP }\end{array} & 26 & 14 & 2 & 5 & 7 & 54 \\ \text { Idioma } & & & & & & \\ \text { Ingles } & 36 & 1 & 6 & 5 & 5 & 53 \\ \text { Español } & 21 & 14 & 0 & 1 & 3 & 39 \\ \text { Tipo de artículo } & & & & & \\ \text { AO } & 37 & 14 & 6 & 1 & 5 & 63 \\ \text { Revisión } & 14 & 1 & 0 & 5 & 3 & 23 \\ \text { CE } & 5 & 0 & 0 & 0 & 0 & 1 \\ \text { Reporte de caso } & 1 & 0 & 0 & 0 & 3 & 64 \\ \text { Colaboración } & 45 & 5 & 6 & 5 & 1 & 37 \\ \text { Colaboración } & 25 & 3 & 3 & 5 & 0 & 16 \\ \text { internacional } & 16 & 0 & 0 & 0 & 1 & 8 \\ \text { USA } & 2 & 2 & 0 & 3 & 0 & 8 \\ \text { España } & 4 & 1 & 3 & 0 & 0 & 4 \\ \text { Brasil } & 3 & 0 & 0 & 1 & & \end{array}$

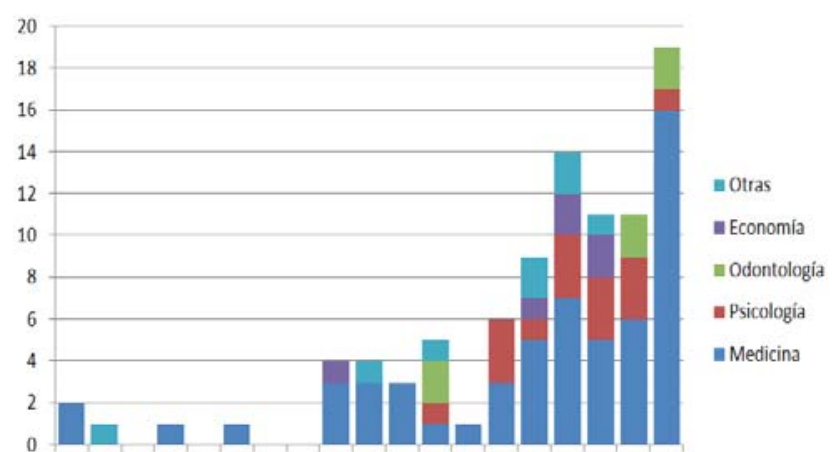

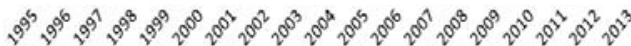

Figura 1.- Producción científica de la Universidad de San Martín de Porres en Scopus, 1995-2013 (N=92).
El 45,6\% de los artículos publicados por la Facultad de Medicina Humana y el $93,3 \%$ de los publicados por la Facultad de Psicología, tuvieron como autor corresponsal a un investigador con filiación USMP. El $57,6 \%$ de los artículos fueron publicados en español, y el $42,4 \%$ en inglés.

El $68,5 \%$ fueron artículos originales. Asimismo, 69,6\% de los artículos fueron considerados "artículos de colaboración", y 40,2\% fueron considerados "artículos de colaboración internacional", con gran participación de USA y España, como se detalla en la tabla 1. 
Se encontraron 118 autores diferentes con filiación USMP, los que publicaron en 70 revistas diferentes (dos de ellas peruanas). Tabla 2.

Tabla 2. Autores con mayor producción científica, y revistas con mayor número de artículos encontrados, al evaluar la producción científica de la Universidad de San Martín de Porres en Scopus, 1995-2013 (N=92).

\begin{tabular}{|c|c|c|}
\hline AUTOR & ARTICULOS & DETALLE \\
\hline César Merino-Soto & 10 & Facultad de Psicología \\
\hline Sixto E. Sánchez & 9 & Facultad de Medicina Humana \\
\hline Ricardo Fujita & 8 & Facultad de Medicina Humana \\
\hline Alberto Salazar Granar & 4 & Facultad de Medicina Humana \\
\hline Luis Carranza & 4 & Facultad de Ciencias Contables, Económicas y Financieras \\
\hline María L. Guevara Fujita & 4 & Facultad de Medicina Humana \\
\hline Berta Loja Herrera & 4 & Facultad de Medicina Humana \\
\hline Eduardo Ticona & 3 & Facultad de Medicina Humana \\
\hline Luz Huaroto & 3 & Facultad de Medicina Humana \\
\hline REVISTA & ARTÍCULOS & DETALLE \\
\hline $\begin{array}{l}\text { Revista Peruana de Medicina } \\
\text { de Experimental y Salud Pública }\end{array}$ & 6 & $\begin{array}{l}\text { Revista del Instituto Nacional } \\
\text { de Salud del Perú }\end{array}$ \\
\hline $\begin{array}{l}\text { Revista de Gastroenterología } \\
\text { del Perú }\end{array}$ & 5 & $\begin{array}{l}\text { Revista de la Sociedad de } \\
\text { Gastroenterología del Perú }\end{array}$ \\
\hline Universitas Psychologica & 3 & $\begin{array}{l}\text { Revista de psicología de la Pontífica } \\
\text { universidad Javeriana }\end{array}$ \\
\hline $\begin{array}{l}\text { International Journal of Molecular } \\
\text { Epidemiology and Genetics }\end{array}$ & 3 & $\begin{array}{l}\text { Revista virtual de acceso libre sobre } \\
\text { genética y epipdemiología molecular }\end{array}$ \\
\hline${ }^{*}$ Revistas peruanas. & & \\
\hline
\end{tabular}

\section{DISCUSIÓN}

Se encontró una tendencia positiva en la producción científica de la USMP, lo cual concuerda con el crecimiento de la producción científica del Perú (15).

Sin embargo, en el año con mayor producción científica (2013) se publicaron apenas 19 artículos: un número reducido para una Universidad que cuenta con dieciocho carreras profesionales, 12 secciones de postgrado, 3 doctorados, más de treinta mil alumnos y 2500 trabajadores entre docentes y administrativos (12). En vista de lo cual resulta necesario mejorar las estrategias utilizadas en favor de la investigación científica.

Visto que una de las grandes limitaciones a las que se enfrenta la investigación científica en el Perú es el escaso financiamiento (16), la USMP debería desplegar esfuerzos tanto para aumentar el presupuesto destinado a esta actividad (4), como para capacitar a sus investigadores en la obtención de fondos concursables nacionales y extranjeros (17).
Entre otras estrategias tenemos: implementar los institutos de investigación, capacitar a docentes e investigadores en temas de redacción y publicación científica (16), establecer sólidas líneas de investigación y exigir a docentes, investigadores y alumnos la publicación de artículos de investigación en revistas indizadas en bases de datos de renombre (18).

De esta manera, la gran masa de estudiantes, podría contribuir enormemente a la producción científica de la USMP, publicando artículos científicos bajo la adecuada tutela de docentes que tengan experiencia y producción científica (19), o publicando sus tesis bajo la modalidad de "titulación por publicación" (20), como se observa en la experiencia de la Universidad Peruana de Ciencias Aplicadas (21).

Asimismo, se puede aprovechar el interés científico de ciertos estudiantes que se reúnen en las Sociedades Científicas Estudiantiles con el fin de lograr publicaciones científicas $(22,23)$. Brindar apoyo a estas sociedades podría multiplicar su producción científica, y contagiar a otros 
estudiantes a dedicarse a estas actividades.

El idioma de publicación influye en la visibilidad del artículo. De esta manera, un artículo publicado en inglés puede perder cierta visibilidad en países de habla hispana, pero tiene muchas más posibilidades de ser descubierto y citado en el resto del mundo (24-26).

Por ello, resulta alentador que el $57,6 \%$ de los artículos evaluados fueron publicados en inglés, y es recomendable continuar incentivando la publicación de artículos en este idioma, así como apoyar a los investigadores que aún no lo dominan, brindando capacitaciones en redacción y publicación científica en inglés.

El $69,6 \%$ de los artículos, fueron realizados en colaboración con autores que tienen filiaciones ajenas a la USMP, y el 40,2\% ha contado con colaboración institucional.

Este es otro punto a favor, pues demuestra la existencia de una red de colaboración interinstitucional que incluso sobrepasa las fronteras nacionales, y permite realizar investigaciones destinadas a revistas de alto impacto $(27,28)$.

Sin embargo, se observa que la mayoría de artículos con colaboración internacional se ha realizado con países que no comparten la realidad peruana (USA, España), por lo cual;se debe tener cuidado de no caer en el neo-colonialismo científico, en el cual los autores principales son extranjeros, y realizan investigaciones que no responden a las necesidades de los países que participan en ellas $(5,29)$.

Al respecto, la Facultad de Medicina Humana de la USMP ha logrado nexos con universidades de prestigio en el exterior para desarrollar cursos de desarrollo para promover la investigación desde el año 2012, y se espera que los graduados de estos cursos utilicen las redes obtenidas para aumentar la producción científica.

La lista de revistas en las que se encontró mayor número de publicaciones, está encabezada por las dos únicas revistas biomédicas peruanas que se encuentran indizadas en Scopus. Resulta necesario que otras revistas peruanas ingresen a esta base de datos, para aumentar la visibilidad y la producción científica de nuestro país.

La USMP se debería preocupar por diseñar y ejecutar estrategias para que sus revistas científicas (como Horizonte Médico de la Facultad de Medicina Humana, o Liberabit de la Facultad de Psicología) sean admitidas en dicha base de datos, lo cual aumentaría su producción científica y el reconocimiento nacional e internacional.

Entre las limitaciones del estudio cabe mencionar que, al evaluar solamente la base de datos Scopus, no se puede tener una aproximación a la producción científica global de la USMP en revistas indizadas en bases de datos regionales o no indizadas.

Otra limitación es que algunos miembros de la USMP pueden no estar colocando en sus filiaciones a Ia USMP, con lo cual dichas publicaciones no han sido contadas en nuestro análisis.

Esto se puede deber a una falta de identificación con la institución, lo cual deberá ser trabajado por los institutos de investigación de la misma.

En conclusión, se evidenció una tendencia al alza en el número anual de publicaciones. Producción científica que debería continuar en desarrollo, por lo cual resulta necesario aplicar estrategias para incentivar y exigir la producción científica en investigadores, docentes y estudiantes de la USMP.

\section{Fuentes de financiamiento}

El presente estudio ha sido autofinanciado.

\section{Conflictos de interés}

Los autores, son egresados de la Facultad de Medicina Humana de la Universidad de San Martín de Porres.

\section{Correspondencia:}

Álvaro Taype-Rondán

Dirección: Calle 22 N 159, Dpto. 102, Urb Corpac, San Borja, Lima, Perú.

Teléfono: 970001517 / 2265586

Correo electrónico: alvaro_athos@hotmail.com 


\section{REFERENCIAS BIBLIOGRÁFICAS}

1. Zhang X, Nan P. The Conception and Practice of Research-Based Teaching in Research Universities. 2014 International Conference on Education Reform and Modern Management (ERMM-14) [Internet]. Atlantis Press; 2014 [citado 24 de junio de 2014]. Recuperado a partir de: http:// www.atlantis-press.com/php/ download_paper.php?id=11211

2. Sedlacek S. The role of universities in fostering sustainable development at the regional level. Jour Clean Prod. 2013;48:7484.

3. Scimago Research Group. SIR Iber Peru 2014 [Internet]. 2014 [citado 25 de junio de 2014]. Recuperado a partir de: http: / / www. scimagoir.com/pdf/iber/SIR\%20lber\%20PER\%202014\%20HE.pdf

4. Romaní F, Cabezas C, Espinoza M, Minaya G, Huaripata J, Ureta JM, et al. Estrategia para fortalecer las capacidades de investigación en salud en universidades públicas regionales: rol del canon y del Instituto Nacional de Salud. Rev Peru Med Exp Salud Publica. 2012;29(4):549-54.

5. Huamaní C, Mayta-Tristán P. Producción científica peruana en medicina y redes de colaboración, análisis del Science Citation Index 2000-2009. Rev Peru Med Exp Salud Publica. 2010;27(3):31525.

6. Sogi C, Perales A, Anderson A, Bravo E. El proceso de producción científica de los investigadores de la Facultad de Medicina, UNMSM. An Fac Med. 2013;63(2):115-24.

7. Barjak F. Research productivity in the internet era. Scientometrics. 2006;68(3):343-60.

8. Falagas ME, Pitsouni El, Malietzis GA, Pappas G. Comparison of PubMed, Scopus, Web of Science, and Google Scholar: strengths and weaknesses. FASEB J. 2008;22(2):338-42.

9. University Rankings, Guides, Forums \& Events | Top Universities [Internet]. [citado 9 de octubre de 2014]. Recuperado a partir de: http:// www. topuniversities.com/

10. Ranking Web of Universities [Internet]. [citado 9 de octubre de 2014]. Recuperado a partir de: http://www.webometrics.info/

11. Ránkings | AméricaEconomía [Internet]. [citado 9 de octubre de 2014]. Recuperado a partir de: http://rankings.americaeconomia. $\mathrm{com} /$

12. USMP - Universidad de San Martín de Porres [Internet]. [citado 8 de octubre de 2014]. Recuperado a partir de: http: / / www.usmp.edu. pe/

13. Facultad de Medicina Humana - Universidad de San Martín de Porres [Internet]. [citado 8 de octubre de 2014]. Recuperado a partir de: http:// www. medicina.usmp.edu.pe/

14. USMP. Reglamento General de la Universidad de San Martín de Porres [Internet]. Lima, Perú: USMP; 2006 [citado 8 de octubre de 2014]. Recuperado a partir de: www.usmp.edu.pe/nuesuniv/pdf/ REGLAMENTO GENERAL USMP.pdf

15. SCImago. SCImago Journal \& Country Rank [Internet]. 2007 [citado 18 de noviembre de 2012]. Recuperado a partir de: http://www. scimagoj r.com
16. Yagui M, Espinoza M, Caballero P, Castilla T, Garro G, Yamaguchi LP, et al. Avances y retos en la construcción del sistema nacional de investigación en salud en el Perú. Rev Peru Med Exp Salud Publica. 2010;27(3):387-97.

17. Garro G, Mormontoy H, Yagui M. Gestión y financiamiento de las investigaciones por el Instituto Nacional de Salud, Perú 2004-2008. Rev Peru Med Exp Salud Publica. 2010;27(3):361-6.

18. Pacheco-Romero J, Huerta D, Galarza C, Ramos-Castillo J. Producción científica de los Institutos y Centro de Investigación de la Facultad de Medicina de la Universidad Nacional Mayor de San Marcos. An Fac Med. 2012;73(2):147-51.

19. Pereyra-Elías R, Huaccho-Rojas JJ, Taype-Rondan A, Mejia CR, Mayta-Tristán P. Publicación y factores asociados en docentes universitarios de investigación científica de escuelas de Medicina del Perú. Rev Peru Med Exp Salud Publica. 2014;31(3):424-30.

20. Taype-Rondán A, Carbajal-Castro C, Arrunategui-Salas G, ChambiTorres J. Limitada publicación de tesis de pregrado en una facultad de medicina de Lima, Perú, 2000-2009. An Fac med. 2012;73(2):153-7.

21. Risco de Domínguez G. Diseño e implementación de un currículo por competencias para la formación de médicos. Rev Peru Med Exp Salud Publica. 2014;31(3):572-81.

22. Taype-Rondán A, Lajo-Aurazo Y, Gutiérrez-Brown R, ZamalloaMasías N, Saldaña-Gonzales M. Aporte de las sociedades estudiantiles en la publicación científica en SciELO-Perú, 20092010. Rev Peru Med Exp Salud Publica. 2011;28(4):691-2.

23. Taype-Rondán A, Bazán-Ruiz S, Valladares-Garrido D. Producción científica de las sociedades científicas de estudiantes de medicina del Perú, 2002-2012. CIMEL. 2013;18(1):23-9.

24. Huamaní C, González-Alcaide G. Publicación de artículos en inglés en revistas médicas en español: ¿Realmente no aumenta el número de citaciones en revistas indexadas? Rev Médica Chile. 2013;141(6):807-8.

25. González-Alcaide G, Valderrama-Zurián JC, Aleixandre-Benavent R. The Impact Factor in non-English-speaking countries. Scientometrics. 2012;92(2):297-311.

26. Kirchik $O$, Gingras $Y$, Larivière $V$. Changes in publication languages and citation practices and their effect on the scientific impact of Russian science (1993-2010). J Am Soc Inf Sci Technol. 2012;63(7):1411-9.

27. Leta J, Chaimovich $\mathrm{H}$. Recognition and international collaboration: the Brazilian case. Scientometrics. 2004;53(3):325-35.

28. Chen TJ, Chen YC, Hwang SJ, Chou LF. International collaboration of clinical medicine research in Taiwan, 1990-2004: a bibliometric analysis. J Chin Med Assoc. 2007;70(3):110-6.

29. Dahdouh-Guebas F, Ahimbisibwe J, Moll RV, Koedam N. Neocolonial science by the most industrialised upon the least developed countries in peer-reviewed publishing. Scientometrics. 2003;56(3):329-43.
Recibido: 29 de Agosto de 2014 Aprobado: 06 de Octubre de 2014 